УДК 66.048.37; 66.046.9; 66.042.886.4

\title{
Т.В. Дьяченко
}

Одесская национальная академия пищевых технологий, Учебно-научный институт холода, криотехнологий и экоэнергетики им. В. С. Мартыновского, ул. Дворянская, 1/3, Одесса, 65082, Украина

\section{ИССЛЕДОВАНИЕ ХАРАКТЕРИСТИК ТЕПЛОМАССООБМЕННОЙ ПОВЕРХНОСТИ НАСАДОЧНЫХ КОЛОНН, ПЕРСПЕКТИВНЫХ ДЛЯ ОБОГАЩЕНИЯ НЕОНОГЕЛИЕ- ВОЙ СМЕСИ}

Одним из главных факторов, определяющих работу насадочных ректификационных колонн, является массообменная поверхность. Представленные исследования посвящены выбору насадки для проектирования аппарата первичного обогащения неоногелиевой смеси. Созданная универсальная экспериментальная установка для изучения процессов ректификации позволила получить основные характеристики четырех насадок. Из исследованных насадок была выбрана лучшая в соответствии с известными требованиями и с учетом маситабного фактора.

Ключевые слова: Ректификация - Неоногелиевая смесь - Насадочная колонна - Массообменная поверхность - Высота единицы переноса

\section{Т.В. Дьяченко}

Одеська національна академія харчових технологій, Навчально-науковий інститут холоду, кріотехнологій та екоенергетики ім. В.С. Мартиновського, вул. Дворянська, 1/3, Одеса, 65082, Україна

\section{ДОСЛІДЖЕННЯ ХАРАКТЕРИСТИК ТЕПЛОМАСООБМІННОЇ ПОВЕРХНІ НАСАДКО- ВИХ КОЛОН, ПЕРСПЕКТИВНИХ ДЛЯ ЗБАГАЧЕННЯ НЕОНОГЕЛІЄВОЇ СУМІШІ}

Одним з головних факторів, щзо визначають роботу насадкових ректифікаційних колон, $\epsilon$ масообмінна поверхня. Представленні дослідження присвячені вибору насадки для проектування апарату первинного збагачення неоногелієвої суміші. Створена універсальна експериментальна установка для вивчення прочесів ректифікачії дозволила отримати основні характеристики чотирьох насадок. Із досліджених насадок була вибрана найкраща у відповідності з відомими вимогами и з урахуванням масмтабного фактору.

Ключові слова: Ректифікація - Неоногелієва суміш - Насадкова колона - Масообмінна поверхня - Висота одинииі переносу

DOI: $10.15673 / 0453-8307.2 / 2015.37236$

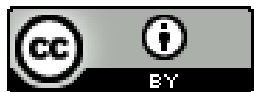

\section{I. ВВЕДЕНИЕ}

Процессы фазовой сепарации широко используются в химических технологиях. Ректификация - это процесс разделения бинарных или многокомпонентных смесей за счет противоточного массо- и теплообмена между паром и жидкостью.

Качество работы ректификационной колонны определяется [1-6]:

- балансом «тепла» и «холода», подводимых к кубу и конденсатору колонны;

- типом используемой массообменной поверхности.

В области низких температур применяют тарельчатые и насадочные ректификационные аппараты. Насадочные колонны более компактны, однако, их использование имеет ограничение. Оно связано с неравномерным распределением пара и жидкости по сечению колонны при диаметрах более 250 мм [3].

\section{ІІ. АНАЛИЗ ЛИТЕРАТУРНЫХ ДАННЫХ И ПОСТАНОВКА ПРОБЛЕМЫ}

Основные типы насадок, используемых в криогенной технике, показаны на рис. 1 и в табл. 1 [1, 6-10].

В современных воздухоразделительных установках (ВРУ) средней и малой производительности в качестве верхней колонны (низкого давления) двухколонного ректифицикационного блока часто применяются аппараты насадочного типа $[6,7]$. Такое решение уменьшает гидравлические потери, снижает габариты установки. Широкое распространение получили регулярные насадки фирмы «Sulzer Chemtech» (рис. 1, a), используемые в ВРУ практически всех производителей. 
В малорасходных колоннах для получения неона высокой чистоты и разделения его изотопов применяют спиральную либо спиральнопризматическую насадку (рис. 1, б, в) [6-9], которая отличается высокой удельной поверхностью теплообмена.

Сетчатая седловидная насадка (рис. 1, г) используется в процессах обогащения тяжелых инертных газов и очистки фреонов [10]. В каждом конкретном случае проектирования насадочной
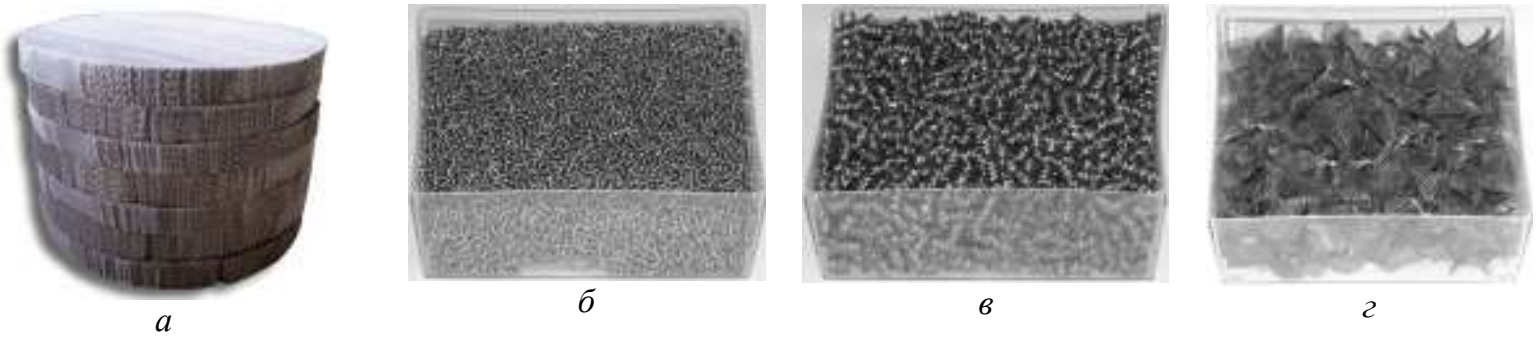

Рисунок 1 - Насадки, используемые в колоннах для сепарации смесей на криогенном уровне температур: $a$-насадки фирмы «Sulzer Cheтtech»; б-спиральная;

в-спирально-призматическая; г - сетчатая седловидная

Таблица 1 - Технические характеристики нерегулярных насадок

\begin{tabular}{|c|c|c|c|c|c|}
\hline Параметр & Формула & \multicolumn{2}{|c|}{ Сетчатая седловидная } & Спиральная & $\begin{array}{c}\text { Спирально- } \\
\text { призматическая }\end{array}$ \\
\hline Материал & - & \multicolumn{2}{|c|}{ Латунь } & Алюминий & Нерж. сталь \\
\hline $\begin{array}{l}\text { Характерные размеры, } \\
\text { мм }\end{array}$ & $a \times b \times d_{\Pi \mathrm{P}}$ & $10 \times 10 \times 0,2$ & $12 \times 12 \times 0,4$ & $3,35 \times 3,34 \times 0,75$ & $3,66 \times 4,24 \times 0,25$ \\
\hline $\begin{array}{l}\text { Свободный объем (по- } \\
\text { ристость), } \mathrm{m}^{3} / \mathrm{M}^{3}\end{array}$ & $\varepsilon=\frac{V_{\mathrm{CB}}}{V_{\Sigma}}(1)$ & 0,935 & 0,969 & 0,673 & 0,894 \\
\hline $\begin{array}{l}\text { Удельная массообмен- } \\
\text { ная поверхность, } \mathrm{m}^{2} / \mathrm{M}^{3}\end{array}$ & $\sigma=\frac{F_{\Pi \mathrm{P}}}{V_{\Sigma}}(2)$ & 1446 & 311 & 1764 & 1701 \\
\hline $\begin{array}{l}\text { Эквивалентный диа- } \\
\text { метр, мм }\end{array}$ & $d_{\ni}=\frac{4 \varepsilon}{\sigma}(3)$ & 2,59 & 12,5 & 1,53 & 2,10 \\
\hline Насыпная масса, кг/м³ & $\rho_{\mathrm{H}}=\frac{m}{V_{\Sigma}}(4)$ & 556 & 565 & 884 & 836 \\
\hline
\end{tabular}

\section{III. РЕЗУЛЬТАТЫ ИССЛЕДОВАНИЯ НАСА- ДОК, ПЕРСПЕКТИВНЫХ ДЛЯ СЕПАРАЦИИ СМЕСИ (Ne-He)- $\mathbf{N}_{2}$}

3.1. Особенности конструкции экспериментального стенда. Универсальный стенд для исследования процессов ректификации (рис. 2) [11-15] был создан на кафедре криогенной техники Одесской национальной академии пищевых технологий (ОНАПТ) в 2009 г. на технической базе предприятия ООО «Айсблик» (Одесса).

В установке предусмотрена возможность замены насадочной части; дискретного наращивания ее высоты $(\Delta H=2$ м) и использования двух видов кубовой секции (с электрическим и жидкостным нагревом), что позволяет исследовать процессы сепарации смесей, в составе которых присутствуют углеводороды.

Кроме ректификационной колонны и систем подачи исходной смеси, а также отвода основного продукта и утилизации отбросных фракций схема включает каскадную систему охлаждения на основе термосифона (рис. 2). В зависимости от решаемой задачи полость 2 заполняется различными веществами-посредниками. Таким образом реализована возможность охлаждения конденсатора на различных температурных уровнях от температуры кипения жидкого азота до практически температуры окружающей среды.

Исследования были проведены для смеси $(\mathrm{Ne}-\mathrm{He})-\mathrm{N}_{2}$ и трех типов насадки: спиральной, спирально-призматической и сетчатой седловидной двух типоразмеров $(10 \times 10 \times 0,2$ мм и $12 \times 12 \times 0,4$ мм, табл. 2) в насадочных секциях высотой 2 м и диаметром 80 мм. Поскольку в состав разделяемой смеси не входят горючие компоненты, был использован куб с электрическим нагревом (см. рис. 2). 
3.2. Методика исследований и результаты экспериментов. Предполагалось, что результаты экспериментов будут использованы в процессе проектирования аппарата первичного концентрирования (АПК) неоногелиевой смеси. По предварительным расчетам диаметр насадочной части АПК составляет 150...200 мм, следовательно, скорость пара будет составлять $0,3 \ldots 0,5 \mathrm{~m} / \mathrm{c}$. Чтобы результаты исследований для указанных насадок можно было масштабировать, в процессе экспериментов поддерживалась скорость движения пара из того же интервала, что и проектируемый аппаpat.

Определение сопротивления сухой насадки. Подготовка колонны включает продувку всех магистралей газообразным азотом с последующим вакуумированием. Далее осуществляли подачу сухого газообразного азота через вентиль $\mathrm{K}_{1}$. Выход азота производится через вентиль $\mathrm{K}_{3}$. Сопротивление сухой насадки было измерено по разнице давлений, измеренных манометрами $\mathrm{P}_{4}$ и $\mathrm{P}_{5}$.

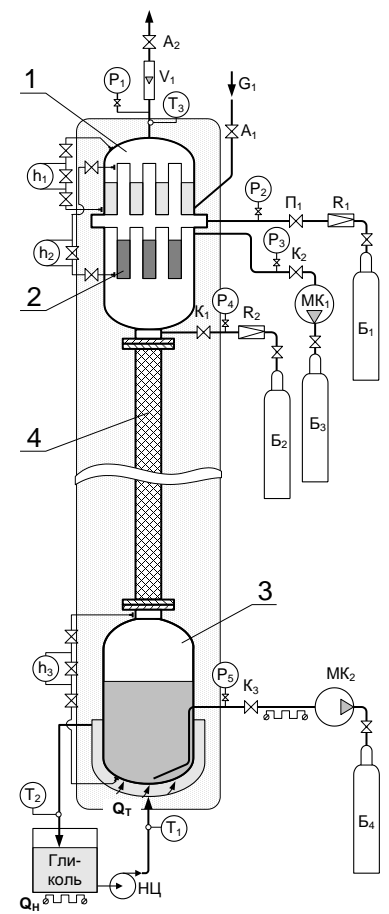

$a$

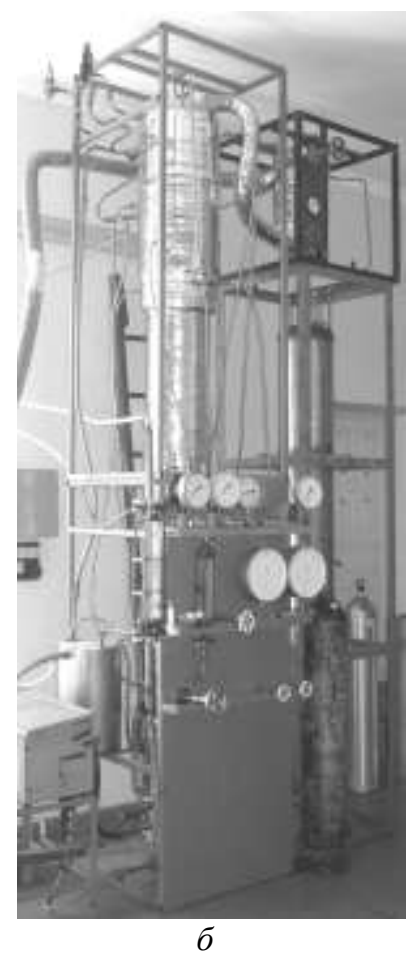

б
Рисунок 2 - Схема (а) и внешний вид (б) экспериментальной установки для исследования процессов ректификаиии: 1-азотная полость; 2 контур промежуточного хладагента; 3 -рабочая полость колонны; 4-массообменная поверхность; ВН-вакуумный насос; $\quad \mathrm{MK}_{1}, \mathrm{MK}_{2}-$ мембранные компрессоры; НЦ-циркуляционный насос; $\quad h_{1}, h_{2}, h_{3}$-указатели уровня; $V_{1}-$ измеритель расхода азота; $Б_{1}-$ баллон с промежуточным хладагентом; $5_{2}-5_{4}-$ баллоны с исходной смесью, газовой и кубовой фракциями

Охлаждение системы. Жидкий азот заливался в полость 1 до уровня 0,2 м по прибору $\mathrm{h}_{1}$. Испытание насадки производится при азотных температурах, поэтому полость хладагента- посредника 2 также заполняли азотом, конденсируя его из баллона $5_{1}$ до уровня 0,5 м по прибору $\mathrm{h}_{2}$ (см. рис. 2).

Заполнение колонны исследуемым веществом производилось путем конденсации из баллона Б до уровня 0,3 м по прибору $\mathrm{h}_{3}$. Модельная смесь в газообразном виде подавалась выше насадки, под конденсатор колонны. Подача прекращалась после заполнения куба колонны до уровня 0,3 м.

Определение теплопритоков $\kappa$ аппарату. В течение 2..3 часов параметры установки поддерживали постоянными, измеряя расход и температуру газообразного азота на выходе из полости 2. Средняя величина теплопритоков составила 63 Вт.

Экспериментальное определение времени наступления равновесных концентраций в режиме без отбора. Для этого осуществлялся подогрев куба нагревателем мощность 2 кВт, конденсатор колонны охлаждался. В процессе эксперимента через каждые 15 минут измерялась концентрация кубовой жидкости и отдувки хроматографом марки 3700 с точностью 0,1\%. Эксперимент считался завершенным, если концентрации не менялась в течение 1 часа.

Определение сопротивления орошаемой насадки производили в процессе сепарации по манометрам $\mathrm{P}_{3}$ и $\mathrm{P}_{4}$.

Измерение удерживающей способности насадки производилось в процессе отогрева колонны. Для этого из куба колонны сливали всю жидкость (азот), а, затем, хладагент из полости посредника и основной хладагент из полости 1 (см. рис. 2). Температура и давление в колонне медленно повышались. Образовавшийся в процессе отогрева пар скачивали компрессором МК в баллон Б 3 до достижения температуры окружающей среды $(280 \ldots 290 \mathrm{~K})$.

Обработка результатов. В процессе обработки результатов определяли объем жидкости, которая испарилась в процессе отогрева; удерживающую способность - объем жидкости на единицу объема насадки (табл. 2).

Определение высоты единицы переноса производили путем стандартных построений для расчета ректификации по методу Мак Кэба и Тиле. При этом расчеты производились для бинарной смеси $\mathrm{Ne}-\mathrm{N}_{2}$. Высота единицы переноса рассчитывалась путем деления высоты насадочной части аппарата на число теоретических ступеней.

Исследования показали, что лучшей из исследованных массообменных поверхностей по ВЕП является спиральная. Однако удельное сопротивление для указанной орошаемой насадки в этом случае выше, чем для остальных. Поскольку этот критерий является определяющим, то лучшим вариантом для разделения смеси ( $\mathrm{Ne}-\mathrm{He})-\mathrm{N}_{2}$ при высоких скоростях пара принята сетчатая седловидная насадка с размерами элементов $10 \times 10 \times 0,2$ мм. Она обеспечивает приемлемую величину высоты единицы переноса и достаточно низкое значение удельного сопротивления слоя при заданных расходных характеристиках. 
Таблица 2 - Технические характеристики насадок в процессе сепарации смеси (Ne-He)- $\mathrm{N}_{2}$

\begin{tabular}{|c|c|c|c|c|c|}
\hline Параметр & Формула & \multicolumn{2}{|c|}{ Сетчатая седловидная } & Спиральная & $\begin{array}{c}\text { Спирально- } \\
\text { призмати- } \\
\text { ческая } \\
\end{array}$ \\
\hline Материал & - & \multicolumn{2}{|c|}{ Латунь } & Алюминий & Нерж.сталь \\
\hline $\begin{array}{l}\text { Характерные разме- } \\
\text { ры насадки, мм }\end{array}$ & $a \times b \times d_{\Pi P}$ & $10 \times 10 \times 0,2$ & $12 \times 12 \times 0,4$ & $\begin{array}{c}3,35 \times 3,34 \times 0 \\
75\end{array}$ & $\begin{array}{c}3,66 \times 4,24 \times 0 \\
, 25\end{array}$ \\
\hline $\begin{array}{l}\text { Удельное сопротив- } \\
\text { ление сухой насадки, } \\
\text { Па/м }\end{array}$ & $\frac{\Delta p}{l}=\xi \frac{\sigma w^{2} \rho_{\Pi}}{8 \varepsilon}$ & 22,5 & 21,8 & 32,3 & 27,0 \\
\hline 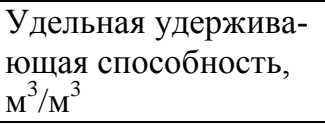 & $H_{0}=\frac{\Delta V_{Ж}}{V_{\Sigma}}$ & 0,161 & 0,158 & 0,138 & 0,165 \\
\hline $\begin{array}{l}\text { Удельное сопротив- } \\
\text { ление орошаемой } \\
\text { насадки, Па/м }\end{array}$ & $\left(\frac{\Delta p}{l}\right)_{\mathrm{OP}}=\xi \frac{\sigma w^{2} \rho_{\Pi}}{8\left(\varepsilon-H_{0}\right)}$ & 48 & 45 & 61 & 53 \\
\hline $\begin{array}{l}\text { Высота единицы пе- } \\
\text { реноса в режиме пол- } \\
\text { ной флегмы и посто- } \\
\text { янного теплового } \\
\text { потока (2 кВт), мм }\end{array}$ & $\mathrm{BE \Pi}=\frac{H_{\mathrm{HAC}}}{N_{\mathrm{CT}}}$ & $50 \ldots 72$ & $62 \ldots 80$ & $45 \ldots 66$ & $47 \ldots 70$ \\
\hline
\end{tabular}

Примечания: $V_{\Sigma}$ - объем, занимаемый насадкой, ${ }^{3} ; V_{\mathrm{CB}}-$ свободный объем, м ${ }^{3} ; F_{\text {Пр }}-$ наружная поверхность насадки, м²; $m$ - масса насадки, кг; $\xi$ - коэффициент сопротивления; $w$ - скорость пара, м/с; $\rho_{\Pi}-$ плотность пара, кг $/ \mathrm{M}^{3} ; \Delta V_{\text {ж }}$ объем жидкости в насадке, ${ }^{3} ; N_{\mathrm{CT}}-$ число теоретических ступеней ректификации для исследуемой смеси и выбранных параметров работы колонны, шт.

\section{IV. ВЫВОДЫ}

В процессе проектирования ректификационного аппарата первичного обогащения неоногелиевой смеси проведены экспериментальные исследования различных массообменных элементов на специально созданной для этого установке. Изучение характеристик трех типов насадок для сепарации неоногелиевой смеси с концентрацией $1 \ldots 2 \%(\mathrm{Ne}-\mathrm{He})$ (сетчатой седловидной двух типоразмеров; спиральной и спиральнопризматической) показало, что оптимальным типом является сетчатая седловидная насадка из проволоки $\varnothing 0,2$ мм с размерами $10 \times 10$ мм. Этот тип насадки при удовлетворении прочих требований обладает низким сопротивлением орошаемой насадки (48 Па), что является критичным для изучаемых скоростей пара $(0,3 \ldots 0,5 \mathrm{~m} / \mathrm{c})$.

Сравнение полученных результатов с расчетными данными и характеристиками насадок, полученными для других смесей (на основе RC318, $\mathrm{SF}_{6}$ и R14), показали хорошую сходимость.

\section{ЛИТЕРАТУРА}

1. Бондаренко В. Л. Криогенные технологии извлечения редких газов [Текст] / В. Л. Бондаренко, Ю. М. Симоненко. - Одесса: Астропринт, 2013. $332 \mathrm{c}$.

2. Головко Г. А. Установки для производства инертных газов [Текст]/ Г.А. Головко. Л.: Машиностроение, Ленингр. отд, 1974. - 383 с.

3. Епифанова В. И. Термодинамические основы разделения воздуха, схемы и аппараты воздухо- разделительных установок [Текст]: в 2 т. Т. 1./ Под ред. В. И. Епифановой, Л. С. Аксельрод. - 2-е изд., перераб. и доп. - М.: Машиностроение, 1973. $-468 \mathrm{c}$.

4. Дьяченко О. В. Проектирование систем охлаждения в технологиях получения чистых газов/ О. В. Дьяченко // Холодильна техніка та технологія. - 2011. - № 5. - С. 15-24.

5. Алексеев В. П. Расчет и моделирование аппаратов криогенных установок [Текст]/ В. П. Алексеев, Г. Е. Вайнштейн, П. В. Герасимов. - Л.: Энергоатомиздат, 1987. - 280 с.

6. Kalbassi M. A. Luquid Distribution from Structured Packings and Distributors under Tilt and Motion Relevant to loating Cryogenic Air Separation Plants / M. A. Kalbassi, B. Waldie, V. White, C. Bell // Procedings $10^{\text {th }}$ International Conference "Cryogenics 2008”. - Praha, Czech Respublic. - 2008. - P. 151158.

7. Arkharov I. Modeling Heat-Mass Transfer Processes on Regular Packing of Distillation Plants / I. Arkharov, E. Navasardyan // Procedings $10^{\text {th }}$ International Conference "Cryogenics 2008". - Praha, Czech Respublic. - 2008. - P. 173-179.

8. Козлов А. В. Рабочие характеристики насадочных колонн установок разделения воздуха [Текст]: автореф. дис. канд. техн. наук: 05.04.03 / МГТУ им. Н. Э. Баумана. - М., 2002. - 16 с.

9. Arkharov A. M. Krypton and Xenon Losses in Low-Pressure Air Separation Rectification Units / A. M. Arkharov, M. Yu. Savinov, V. L. Bondarenko, A. S. Bronshtein // Chemical and Petroleum Engineering. - 2003. - Vol. 39. - Issue 11-12. - P. 725-730. doi: 10.1023/b:cape.0000017619.28195.7e. 
10. Бондаренко В. Л. Промышленные установки для комплексной очистки галокарбонов / В. Л. Бондаренко, О. В. Дьяченко, А. В. Егоров, Ю. М. Симоненко и др. // Холодильна техніка та технологія. - 2007. - № 5 (109). - С. 30-33.

11. Бондаренко В. Л. Многоцелевая установка для исследования процессов ректификации при $T=80 \ldots 300$ К / В. Л. Бондаренко, Т. В. Дьяченко // Humboldt-Kolleg "Sciences, Engineering, and Humanities for the Energy World". - Одесса. - 2009. C. 66-67.

12. Bondarenko V. L. Experimental study of separation processes on packed columns / V. L. Bondarenko, T. V. D'yachenko,

O. V. D'yachen-ko, Yu. M. Simonenko // Chemical and Petroleum Engineering. - 2009. - Vol. 45. - Issue 9-10. - P. 625-626. doi: 10.1007/s10556-010-9227-7.

13. Бондаренко В. Л. Многоцелевая насадочная колонна для изучения процессов сепарации многокомпонентных смесей / В. Л. Бондаренко, T. В. Дьяченко, О. В. Дьяченко // IV Межд. научно-технической конференции «Низкотемпературные и пищевые технологии в XXI веке». Материалы конференции. - Санкт-Петербург. - 2009. C. 340-341.

14. Бондаренко В. Л. Энергетическое обеспечение процессов сепарации в производстве редких и технических газов / В.Л. Бондаренко, Т. В. Дьяченко // Сборник трудов международной научной конференции «Инновационные разработки в области техники и физики низких температур». Москва. - 2010. - С. 3-5.

15. Дьяченко Т. В. Отримання неону та гелію 3 низькопотенційних газових сумішей методом фазової сепарації [Текст]: дисс. канд. техн. наук: 05.14.06 / ОДАХ. - Одеса, 2011. - 22 с.

\section{T.V. Diachenko}

Odessa National Academy of Food Technologies, Educational and Research Institute of Refrigeration, cryotechnology and Ecoenergetics named after V.S. Martynovskiy, 1/3, Dvoryanskaya Str., Odessa, 65082

\section{RESEARCH OF PERSPECTIVE FOR NEON-HELIUM MIXTURE ENRICHMENT HEAT AND MASS NOZZLE COLUMNS SURFACE CHARACTERISTICS}

One of the main factors determining nozzle rectifying columns operation is the mass exchange surface. The presented researches focus on the choice of nozzle design unit for neon helium mixture primary enrichment. Created universal experimental setup for studying the processes of rectification allows obtaining the basic characteristics of four nozzles: spiral, spiral-prismatic and two types of mesh saddle $(10 \times 10 \times 0,2 \mathrm{~mm}$ and $12 \times 12 \times 0,4 \mathrm{~mm})$.

From the studied nozzles according to known requirements and taking into consideration a largescale factor the best mesh saddle nozzle from a wire with a diameter 0,2 $\mathrm{mm}$ and the sizes $10 \times 10 \mathrm{~mm}$ was chosen. This type of nozzle has low resistance of the irrigated nozzle (48 Pa) that is critical for the studied steam speeds $(0,3 \ldots 0,5 \mathrm{~m} / \mathrm{s})$.

Keywords: Rectification - Neon-Helium Mixture - Nozzle Column - Heat and Mass-exchange Surface - Transfer Unit Height.

\section{REFERENCES}

1. Bondarenko V. L., Simonenko Yu. M. 2014. Cryogenic Technologies of Rare Gases Extraction. Odessa: Astroprint. $-312 \mathrm{p}$.

2. Golovko G. A. 1974. Installations For Production of Inert Gases. - Leningrad: Mashinostroenie, Leningradskoe otdelenie - 383 p. (in Russian)

3. Thermodynamic Bases of Air Separation, Schemes and Devices of Air Separation Installations. 1973. Ed. V. I. Epifanova, V. I. Akselrod. - Moscow: Mashinostroenie. -468 p. (in Russian)

4. Diachenko O.V. 2011. Design of Refrigerating Systems in Technologies of Pure Gases Receiving. Kholodylna Tekhnika ta Tekhnologiya [Refrigeration Engineering and Technology], No. 5, 15-24. (in Russian)

5. Alekseev V. P., Vajserasimov P. V. 1987. Calculation and Modeling of Cryogenic Installations Devices. - Leningrad: Energoatomizdat. - 280 p. (in Russian)
6. Kalbassi M. A.,
Waldie B.
White V.

Bell C. 2008. Luquid Distribution from Structured Packings and Distributors under Tilt and Motion Relevant to loating Cryogenic Air Separation Plants. Procedings $10^{\text {th }}$ International Conference "Cryogenics 2008”. - Praha, Czech Respublic. - P. 151-158.

7. Arkharov I., Navasardyan E. 2008. Modeling Heat-Mass Transfer Processes on Regular Packing of Distillation Plants. Procedings $10^{\text {th }}$ International Conference "Cryogenics 2008". - Praha, Czech Respublic. -P. 173-179.

8. Kozlov A. V. 2002. Calculation and Modeling of Cryogenic Installations Devices. Abstract of dissertation for the degree of Candidate of Science (engineering): 05.04.03. MGTU n.a. N. E. Bauman. - Moscow. - 16 p. (in Russian)

9. Arkharov A. M., Savinov M. Yu., Bondarenko V. L., Bronshtein A. S. 2003. Krypton and Xenon Losses in Low-Pressure Air Separation Rectification Units. Chemical and Petroleum Engineering, Vol. 39, Iss. 11-12, 725-730. doi: 10.1023/b:cape.0000017619.28195.7e.

10. Bondarenko V. L., Diachenko O. V., Ego- 
rov A. V., Simonenko Yu. M. 2007. Industrial Plants of Fluorocarbons Purification. Kholodylna Tekhnika ta Tekhnologiya [Refrigeration Engineering and Technology], No.5 (109), 30-33. (in Russian)

11. Bondarenko V. L., Diachenko T. V. 2009. Multipurpose Plant for Research Processes of Rectification at $T=80 \ldots 300 \mathrm{~K}$. Humboldt-Kolleg «Sciences, Engineering, and Humanities for the Energy World». Odessa. - P. 66-67. (in Russian)

12. Bondarenko V. L., D'yachenko T. V., D'yachenko O. V., Simonenko Yu. M. 2009. Experimental Study of Separation Processes on Packed Columns. Chemical and Petroleum Engineering, Vol. 45, Iss. 9$10,625-626$. doi: $10.1007 / \mathrm{s} 10556-010-9227-7$.

13. Bondarenko V. L., Diachenko T. V., Diachenko O. V. 2009. Multi-purpose Packed Column for Studying the Separation Processes of Multicomponent Mixtures. IV International Scientific-technology Conference "Low Temperature Technologies in XXI Century”. - Saint-Petersburg. - P. 340-341. (in Russian)

14. Bondarenko V. L., Diachenko T. V. 2010. Power Supply of the Separation Processes in the Rare and Technical Gases Production. Proceeding International Scientific Conference «Innovative Development in the Field of Equipment and Physics of Low Temperatures». - Moscow. - P. 3-5. (in Russian)

15. Diachenko T. V. 2011. Neon and Helium Reception From Low Potential Gas Mixtures With the Phase Separation Method. Dissertation for the degree of Candidate of Science (engineering): 05.14.06. OSAR. - Odessa. - 22 p. (in Russian)

Отримана в редакції 16.01.2015, прийнята до друку 03.03.2015 